\title{
A Simplified Computational Method for Overall Risk Probability in Probabilistic Health Risk Assessments of Environmental Pollutants
}

\author{
Zhang Wenmei ${ }^{1 a}$,Qiming J. Yu ${ }^{2 b}$, Qiming Cao ${ }^{2 b}$,Des W. Connell ${ }^{2 b}{ }^{1}$ Electric \\ Engineering Dept. of Guangdong AIB Polytechnoic College, Guangzhou. China \\ ${ }^{2}$ Griffith School of Engineering, Griffith University, Nathan Campus, Brisbane, \\ Queensland 4111, Australia.'wenmeizhang@sohu.com, bimmy.yu@griffith.edu.au
}

Keywords: Health risk assessment, overall risk factor, overall risk probability, probabilistic risk characterization.

\begin{abstract}
In the assessment of health risks of environmental pollutants, both conventional methods and probabilistic methods based on the use of cumulative probability plots have been used. Probabilistic risk assessment has the advantage that the statistical distributions of both the exposure data set and the adverse effect data set are taken into account in risk characterization. In addition, the method of overall risk probability (ORP) has been used to quantify the overall risk probability of adverse effects for the population samples. The disadvantage is that many more data points are needed to obtain the cumulative probability plots and the calculation of the ORP from the probability plots is more complicated. In this paper, a simplified computational method for the estimation of the ORP was developed. In this method, an overall risk factor (ORF), as an estimated value of the ORP value, was obtained from the mid-point of an exposure exceedence curve. The ORF can also be obtained from an inversed cumulative probability plot of the exposure curve, thus eliminating the needs for the use of the exposure exceedence curve and the numerical integration of the exceedence curve in ORP computation. The use of ORF method was illustrated with a practical example and the results indicated that the values of the ORF and ORP are about the same.
\end{abstract}

\section{Introduction}

Health risk assessment of environmental pollutants is important in many aspects of environmental management. Examples of such risk assessment include the assessments of human health risks of chlorinated disinfection by-products in drinking water (Hamidin et al. 2008), organic contaminants in indoor air (Sofuoglu et al. 2011), and endocrine disrupting chemicals in water recycling schemes (Cao et al. 2010). Health risk assessment for environmental pollutants has a number of specific difficulties that need to be considered. These include long exposure times up to life times, chronic toxicity effects from low levels of pollutants, and large variations and different statistical distributions in both exposure and dose-response data. In health risk assessment of toxicants, many methods have been used to evaluate and quantify the adverse effects of the toxicants. These methods can be divided into conventional non-probabilistic methods and probabilistic based methods, based on whether the statistical distributions of the exposure and/or adverse effects data are taken into account. Probabilistic methods of risk assessment are relatively newer methods for the evaluation and quantification of health risks from exposure to toxicants (Bosgra et al. 2007; Bosgra et al. 2009; Carriger and Rand 2008; Johnston and Snow 2007; Jager et al. 2001; Thompson and Graham 1996). Risk assessment is based on the use of cumulative 
probability plots of both the exposure and adverse effect (dose response) data sets on the same graph.The characterization and quantification of the health risk in probabilistic risk assessment can be achieved with two approaches. The first approach is based the calculation of a hazard quotient (HQ). In this approach, a margin of safety can also be calculated as the inverse of the hazard quotient. The second approach is based on the calculation of the overall risk probability (ORP) for adverse effect in the sample population. The HQ method is a single point method, while the ORP method is a multiple point method, taking into the statistical distributions of both the exposure data set and the adverse effect data into account. However, the ORP method requires the construction of an exposure exceedence curve. Numerical integration of the exceedence curve is also needed to calculate the value of the ORP. Therefore, numerical analysis for the ORP method is much more complicated. The purpose of this paper was to develop a simplified computational method for the estimation of the ORP, thus making it much easier to use the ORP method in health risk assessment. In the simplified method, an overall risk factor (ORF), as an estimated value of the ORP value, was obtained from the midpoint of an exposure exceedence curve. It was further developed that the ORF can be obtained from an inversed cumulative probability plot of the exposure curve, thus eliminating the needs for the use of the exposure exceedence curve and the numerical integration of the exceedence curve in ORP computation.

\section{Method of Hazard Quotient (HQ)}

In a risk assessment with the probabilistic approach, the risk characterization and quantification are based on the cumulative probability charts for both exposure and adverse effects plotted on the same graph (US EPA 2001; Solomon et al. 2000). These are determined from various experimental measurements. A schematic example is given in Figure 1. The x-axis in Figure 1 is either dose or concentration as the measure of exposure to the toxicant. Logarithm scale is used. The y-axis represents the cumulative probability of the exposure and effects.

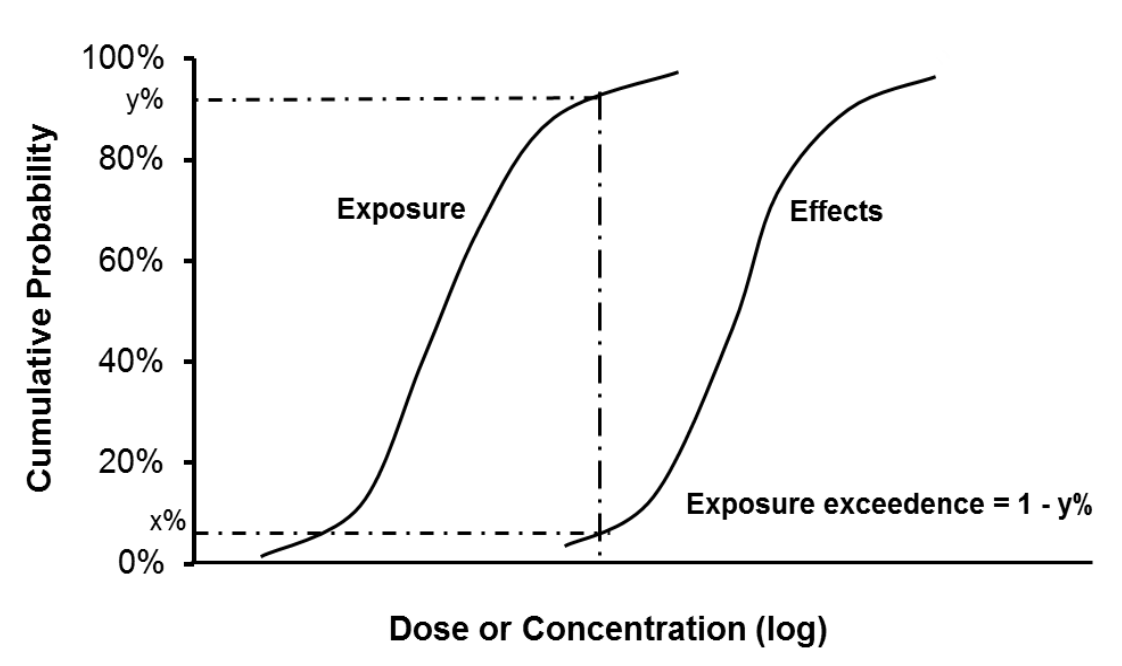

Figure 1 Cumulative probability charts used for calculation of exceedence curves. $\mathrm{x} \%$ represents the percentage of sample affected at a given dose or concentration, y\% represents the cumulative probability of exposure, and 1-y\% represents the exposure exceedence.

Risk characterization and quantification by the probabilistic approach is usually achieved through a comparison between the cumulative probability curves for exposure and effects. Under typical conditions, the exposure curve is on the left hand side of the effect curve, and the closeness 
or overlap between the two curves provides a qualitative indication of the relative degree of the health risk. A closer overlap between the two curves indicates a higher risk. On the other hand, if the two curves are far apart from each other and do not overlap, it can be said that the risk is low or negligible. To obtain a quantitative comparison, a hazard (or risk) quotient (HQ) can be calculated. For example, $\mathrm{HQ}_{95 / 5}$ is defined as the ratio of the exposure dose (or concentration) at 95\% cumulative probability on the exposure curves to that at $5 \%$ cumulative probability at the effect curve (Cao et al. 2011). A reference point of $\mathrm{HQ}_{95 / 5}=1$ corresponds to a risk probability of $1 / 20$, or $5 \%$. Similarly, a reference $\mathrm{HQ}_{50 / 50}=1$ represents a risk probability of $50 \%$. This situation would also indicate that the two cumulative probability curves are largely overlapped with each other over the same dose intervals. However, while the HQ method is a simple method for risk quantification and comparison, because it is only a single point method, the statistical distributions of the cumulative probability curves are not taken into account. The HQ may not represent the true risk characteristics of the system, in particular when the distributions of exposure and effects data do not follow the normal distribution or have different slopes (Cao et al., 2011).

\section{Method of Overall Risk Probability (ORP)}

Recently a new method of overall risk probability (ORP) was developed to quantify the health risks from the two cumulative probability plots (Cao et al. 2011). In this method, an ORP for the adverse health effect is calculated. This method overcomes the nature of a single point evaluation used in the HQ method and provides a straight-forward probability measure for the health risks present in the system. When compared to the HQ method, the ORP method has a number of advantages. The ORP method provides a simple probability measure that can easily compare the relative risks of individual compounds. It is also a multiple-point method such that the statistical distributions of both the exposure and effect curves are properly taken into account. The ORP method also has the advantage that it can quantify mixture effects of either independent or interacting mixtures (Yu et al., 2012).

From the cumulative probability curves as shown in Figure 1, for each point on the effect curve, with the percentage of sample affected identified as $\mathrm{x} \%$, a corresponding exposure probability value can be obtained from the exposure curve as identified by $y \%$ at the same dose or concentration. The exposure exceedence value is then calculated as $1-y \%$. When the exposure exceedence value $1-y \%$ is plotted against the percentage of sample affected, an exposure exceedence curve is obtained. This is illustrated in Figure 2. The exposure exceedence curves can be used to characterize the health risk of the system as represented by the cumulative probability charts. The origin of an exposure exceedence chart represents zero risk and any deviation from the origin represents increasing risks. The higher the exceedence curve, the higher the risk. Quantitatively, the area underneath the exposure exceedence curve represents the overall risk probability of the adverse effects (Cao et al. 2011). This area can be obtained through numerical integration of the exceedence curve.

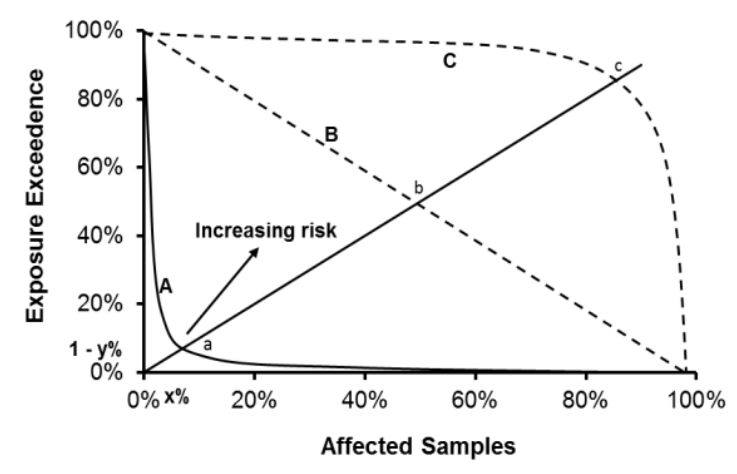


Figure 2 Exceedence curves used for calculation of overall risk probabilities. $x \%$ represents the percentage of sample affected and $1-\mathrm{y} \%$ represents the exposure exceedence. A, B, and C are three separate exceedence curves with increasing risk probabilities and a, b, and $\mathrm{c}$ are the intersection points (mid-points) of the exceedence curves with the 45 degree diagonal line.

\section{A Simplified Computational Method for ORP}

In order to simplify the computational procedures of the ORP method, a 45 degree diagonal line is drawn from the origin in Figure 2, and the intersection points of the diagonal line with the exceedence curves, or the mid-points of the exceedence curves are identified as a, b, a in Figure 2. The probability values of these mid-points represent the approximate values of the areas underneath each of the exceedence curves. Therefore, these values present the estimated valuations of the ORPs. In order to distinguish these values from the ORP values calculated through numerical integration, these values are here referred to as overall risk factors (ORF).

As the diagonal line in Figure 2 represents the equal probability values of the $x$-axis and the $y$-axis, and the $y$-axis values are the exceedence values calculated from the 1-y\% values, the mid-points in the exceedence curves can also be identified as the intersection points between the effect curve and an inversed exposure curve, which is calculated as the $1-y \%$ values of the exposure curve. This is illustrated as the dashed line in Figure 3. Therefore, the ORP values can be readily obtained as shown in Figure 3, without the use of the exceedence curves in Figure 2. This greatly simplifies the computational procedures in the calculations of the ORF values.

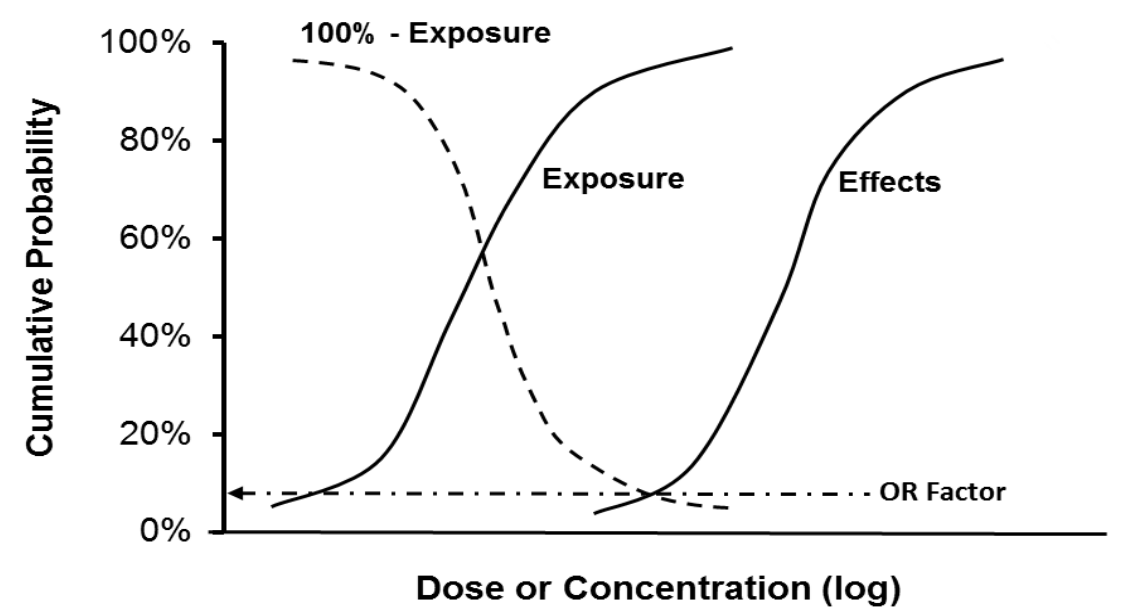

Figure 3 Cumulative probability plots used for calculation of overall risk factor (OR factor), The dashed line is the inversed exposure curve as represented by the values of $1-y \%$.

\section{A Practical Example of ORP and ORF Evaluation}

As an example, the ORP and the ORF of two endocrine disrupting chemicals (EDCs) in aquatic environment were evaluated and given below. The ORP method has been used in the assessment of the adverse effect of significant level of vittellogenin induction in fish exposed to $17 \beta$-estadiol (E2) and 17 $\alpha$-ethinylestradiol (EE2) in fresh water receiving treated wastewater effluents (Cao et al., 2011). The ORPs obtained for E2 and EE2 in the study are $\left(\mathrm{ORP}_{\mathrm{E} 2}=0.081\right.$ or $\left.8.1 \%\right)$ and $\left(\mathrm{ORP}_{\mathrm{EE} 2}=0.27\right.$ or $\left.27 \%\right)$. In this paper, the same sets of data were used to evaluate the ORF values of $\mathrm{E} 2$ and $\mathrm{EE} 2$, and the values are $\left(\mathrm{ORF}_{\mathrm{E} 2}=0.08\right.$ or $\left.8 \%\right)$ and $\left(\mathrm{ORF}_{\mathrm{EE} 2}=0.27\right.$ or $\left.27 \%\right)$. The 
numerical values obtained by the two methods are practically the same, thus validating the usefulness of the new method.

\section{Conclusions}

A new method of overall risk factor (ORF) was developed as a simplified computational method for the evaluation of the overall risk probability (ORP) in probabilistic risk characterization. In this method, an ORF was obtained from the midpoint of an exposure exceedence. The ORF can also be obtained from an inversed cumulative probability plot of the exposure, thus eliminating the needs for the use of the exposure exceedence curve and the numerical integration of the exceedence curve in ORP computation. A practical numerical example validated that the values of the ORF and ORP are about the same and the ORF method can be used to quickly estimate the overall health risks of environmental pollutants.

\section{References}

Bosgra S, van der Voet H, Boon P, Muller AK, Bos P, Slob W (2007) An integrated framework for probabilistic cumulative risk assessment of chemicals in food. Toxicology Letters 172:S100-S101

Bosgra S, van der Voet H, Boon PE, Slob W (2009) An integrated probabilistic framework for cumulative risk assessment of common mechanism chemicals in food: An example with organophosphorus pesticides. Regulatory Toxicology Pharmacology 54(2):124-133

Cao Q, Yu Q, Connell DW (2010) Fate simulation and risk assessment of endocrine disrupting chemicals in a reservoir receiving recycled wastewater. Science of the Total Environment 408 (24):6243-6250

Cao Q, Yu Q, Connell DW (2011) Health risk characterisation for environmental pollutants with a new concept of overall risk probability. Journal of Hazardous Materials 187 (1-3):480-487

Carriger JF, Rand GM (2008) Aquatic risk assessment of pesticides in surface waters in and adjacent to the Everglades and Biscayne National Parks: II. Probabilistic analyses. Ecotoxicology 17 (7):680-696

Hamidin N, Yu QJ, Connell DW (2008) Human health risk assessment of chlorinated disinfection by-products in drinking water using a probabilistic approach. Water Research 42 (13):3263-3274

Jager T, den Hollander HA, van der Poel P, Rikken MGJ, Vermeire T (2001) Probabilistic environmental risk assessment for dibutylphthalate (DBP). Human and Ecological Risk Assessment 7 (6):1681-1697

Johnston JJ, Snow JL (2007) Population-based fish consumption survey and probabilistic methylmercury risk assessment. Human and Ecological Risk Assessment 13 (6):1214-1227

Sofuoglu SC, Aslan G, Inal F, Sofuoglu A (2011) An assessment of indoor air concentrations and health risks of volatile organic compounds in three primary schools. International Journal of Hygiene and Environmental Health 214 (1):38-46

Solomon K, Giesy J, Jones P (2000) Probabilistic risk assessment of agrochemicals in the environment. Crop Protection 19 (8-10):649-655

Thompson KM, Graham JD (1996) Going beyond the single number: Using probabilistic risk assessment to improve risk management. Hum Ecol Risk Assess 2 (4):1008-1034

US EPA (2001) Risk Assessment Guidance for Superfund: Volume III - Part A, Process for Conducting Probabilistic Risk Assessment. Available at: www.epa.gov/superfund/RAGS3A/index.htm

Yu QJ, Cao Q, Connell DW (2012) An overall probability based method for qunatification of synergistic and antagonistic effects in health risk assessment for mixtures: Theoretical concenpts. Environmental Science and Pollution Research, in press. 